\title{
Cervical carcinoma-associated fibroblasts are DNA diploid and do not show evidence for somatic genetic alterations
}

\author{
Willem Ernst Corver • Natalja Tatjana ter Haar • \\ Gert Jan Fleuren • Jan Oosting
}

Accepted: 5 September 2011 /Published online: 1 November 2011

(C) The Author(s) 2011. This article is published with open access at Springerlink.com

\begin{abstract}
Background Cancer-associated fibroblasts (CAFs) have been recognized as important contributors to cancer development and progression. However, opposing evidence has been published whether CAFs, in addition to epigenetic, also undergo somatic genetic alterations and whether these changes contribute to carcinogenesis and tumour progression. Methods We combined multiparameter DNA flow cytometry, flow-sorting and $6 \mathrm{~K}$ SNP-arrays to study DNA aneuploidy, \% S-phase, loss of heterozygosity (LOH) and copy number alterations (CNAs) in cervical cancerassociated stromal cell fractions $(n=57)$ from formalinfixed, paraffin-embedded (FFPE) samples. Tissue sections were examined for the presence of CAFs. Microsatellite analysis was used to confirm $\mathrm{LOH}$ findings.

Results Smooth muscle actin and vimentin immunohistochemistry verified the presence of CAFs in all cases tested. However, we found no evidence for DNA aneuploidy, somatic genetic alterations in the vimentin-positive stromal cell fractions of any samples, while high frequencies of DNA content abnormalities (43/57) and substantial numbers of CNAs and $\mathrm{LOH}$ were identified in the keratinpositive epithelial cell fractions. LOH hot-spots on chromosomes $3 p, 4 p$ and $6 p$ found were confirmed by microsatellite analysis.

Conclusion From our study we conclude that stromal cell fractions from cervical carcinomas are DNA diploid, have a genotype undistinguishable from patient-matched normal
\end{abstract}

Electronic supplementary material The online version of this article (doi:10.1007/s13402-011-0061-5) contains supplementary material, which is available to authorized users.

W. E. Corver $(\bowtie) \cdot$ N. T. ter Haar · G. J. Fleuren · J. Oosting Department of Pathology, Leiden University Medical Center, Building 1, L1-Q, P.O. Box 9600, Leiden 2300 RC,

The Netherlands

e-mail: w.e.corver@lumc.nl tissue and are genetically stable. Using flow cytometry and SNP-arrays, stromal genetic changes do not seem to play a role during cervical carcinogenesis and progression. In addition, the stromal cell fraction of cervical carcinomas can be used as reference allowing large retrospective studies of archival FFPE tissues for which no normal reference tissue is available.

Keywords Cervical cancer- Formalin-fixed, paraffinembedded . Carcinoma-associated fibroblast . Vimentin . Smooth-muscle actin - Fluorescence activated cell sorter. SNP-array

\section{Introduction}

A major cellular component of the stroma of epithelial tumours is the carcinoma-associated fibroblast (CAF). Several studies demonstrated that CAFs interact with the tumour cells and play an important role during tumour progression and metastatic spread, and harbour biological characteristics distinct from normal fibroblasts of the same organ $[1,2]$. Isolates from CAFs strongly promote tumour growth and angiogenesis through secretion of stromal cellderived factor $1 \alpha(\mathrm{SDF}-1 \alpha)$ [3]. CAFs resemble myofibroblasts and express vimentin and $\alpha$-smooth muscle actin $(\alpha$ SMA). It is proposed that CAFs originate from bone marrow derived mesenchymal stem cells $[4,5]$.

Stromal epigenetic alterations can have clinical consequences. In oral squamous cell carcinoma it was demonstrated that myofibroblastic stromal SMA expression identified a group of patients with aggressive tumours [6]. Lim and co-workers used cultured fibroblast from normal oral mucosa, epithelial dysplastic tissue and cancerous tissue and identified gene expression profiles that reflected different stages of tumour progression [7]. Amongst involvement of the TNF- $\alpha$ and TGF- $\beta$ pathway, ACTA2, 
a gene encoding for $\alpha$-SMA, was highly expressed. Furthermore, expression signatures from breast cancer strongly associated with clinical outcome [8].

Also cervical cancer stromal cell fractions have been studied at the expression level using micro-arrays. In response to the progression of pre-cancerous stages of the epithelial cell fraction from CIN I to CIN III the underlying stromal cells alter their expression pattern [9, 10]. During CIN III/squamous cell carcinoma transition a pro-invasive gene signature was identified.

The studies mentioned above emphasize an important role for stroma and CAFs particularly during tumour development and progression, and lately reviewed by Allen, et al. [11]. Beside epigenetic changes genetic changes, e.g. frequent copy number alterations (CNAs), loss of heterozygosity ( $\mathrm{LOH}$ ), have been described in CAFs from bladder cancer, breast cancer [12,13], colon cancer [14] and in colitis ulcerosa [15], attributing an important role of these altered fibroblasts during carcinogenesis and tumour evolution.

Whether cervical cancer stroma fibroblasts also undergo somatic genetic alterations has not been investigated and it remains unclear whether these genetic changes, when present, contribute to the development and sustainment of the tumour. This implies that cervical CAFs have a normal genotype and only undergo epigenetic alterations or that cervical CAFs might accumulate somatic genetic alterations dissimilar from the alterations found in the epithelial cell fraction of cervical cancer. Knowledge on the genetic status of cervical CAFs might have consequences for tumour behaviour and therapy, and the general understanding of this common type of cancer.

In the present study we investigated somatic genetic alterations and DNA content of stromal cell fractions derived from FFPE archival cervical carcinomas. Preceding detection of chromosomal aberrations by $6 \mathrm{~K}$ GoldenGate SNP-array, a highly suitable platform for detection of CNAs and LOH in FFPE tissues [16, 17], we used FACS to obtain pure stromal and epithelial tumour subpopulations [18]. Patient-matched normal endometrium was used as reference. Through our new approach we were able to determine DNA content, genotype and genomic status of the stromal and the epithelial fractions in FFPE archival cervical carcinomas in parallel.

\section{Materials and methods}

\subsection{Tissue samples and dissociation}

Cervical carcinomas were obtained from the FFPE-tissue bank of the Department of Pathology, Leiden University Medical Center (LUMC), Leiden, the Netherlands. Samples were handled according to the medical ethical guidelines described in the Code Proper Secondary Use of Human Tissue established by the Dutch Federation of Medical Sciences (www.federa.org).

Since detection of DNA aneuploidy by flow cytometry amongst other factors depends on the coefficient of variation $(\mathrm{CV})$ of the $\mathrm{G}_{0} \mathrm{G}_{1}$ population and the percentage of DNA aneuploid tumour cells in the sample [19], we selected 57 samples from a large series of FFPE cervical carcinomas showing DNA histograms with a $\mathrm{CV}$ of the keratin-positive $\mathrm{G}_{0} \mathrm{G}_{1}$ population of $6.0 \%$ or lower, without a priori knowledge of the ploidy status of the stromal cell fractions.

Paraffin sections taken from all samples were H\&E stained and reviewed by a pathologist (GJF). The tumour nodule, including the intra-tumour stroma, was marked on the H\&E section using a pencil. Preceding flow cytometric processing, carcinoma tissue was enriched for carcinoma and carcinoma-associated stroma by trimming the normal epithelium and/or superfluous stromal tissue and smooth muscle tissue using surgical knifes and the marked-up H\&E section as a guide. This way the workup of stromal cells outside the tumour nodule was prevented.

Cell suspensions were prepared, as described in detail elsewhere [20]. In short, from 57 plus one (example with poorly differentiated tumour cells) cervical carcinomas and matching lymph nodes and/or endometrium six to ten $60 \mu \mathrm{m}$ sections from each paraffin block were taken. After collecting the thick section, a $4 \mu \mathrm{m}$ section was taken and H\&E stained for reviewing the presence of tumour tissue. The thick tissue sections were processed for flow cytometry. In short, sections were dewaxed and submerged in citrate buffer for antigen retrieval at $80^{\circ} \mathrm{C}$ for $60 \mathrm{~min}$. Next, sections were dissociated using a mixture of collagenase/dispase at $37^{\circ} \mathrm{C}$. Then cells were harvested, washed, counted and stored on ice until further use.

\subsection{Immunocytochemistry of cell suspensions}

Immunocytochemistry was performed as described earlier [20], with minor modifications [21]. In short, five million cells were incubated with $200 \mu \mathrm{l}$ of monoclonal antibody mixture in $\mathrm{PBS} / 1 \% \mathrm{BSA} / 0.05 \%$ Tween 20 (PBATw) containing anti-keratin MNF116 (Cat. \# 0821, DAKO, Glostrup, Denmark), working concentration $10 \mu \mathrm{g} / \mathrm{ml}$, antikeratin AE1/AE3 (Cat. \# MAB3412, Millipore-Chemicon, Billerica, MA), working concentration $25 \mu \mathrm{g} / \mathrm{ml}$ PBATw and anti-vimentin V9-2b (diluted 1:5) (Antibodies for Research Applications BV, Gouda, The Netherlands, http://www.abforresearch.nl/) overnight at $4^{\circ} \mathrm{C}$. The next day, cells were washed twice with ice-cold PBATw and centrifuged at $750 \times g$ for $5 \mathrm{~min}$ at $4^{\circ} \mathrm{C}$. Then, cells were incubated with $200 \mu \mathrm{l}$ premixed FITC- or RPE-labelled 
secondary reagents [Goat $\mathrm{F}(\mathrm{ab} 2)^{\prime}$ anti-mouse IgG1-FITC and goat $\mathrm{F}(\mathrm{ab} 2)^{\prime}$ anti-mouse IgG2b-RPE (Southern Biotechnology Associates, Birmingham, AL), both diluted 1:100 in PBATw.] After 30 min on ice, cells were washed twice with ice-cold PBATw and incubated with 1,000 $\mu \mathrm{l}$ NST DNA staining solution containing $50 \mu \mathrm{M}$ DAPI (Sigma Aldrich) [22]. Cells were kept at room temperature for $30 \mathrm{~min}$, followed by an overnight storage at $4^{\circ} \mathrm{C}$. To reduce unwanted cell loss, compensation controls contained one fifth of the concentration of primary antibodies used for labelling samples for sorting. DAPI concentration was decreased to $10 \mu \mathrm{M}$.

\subsection{Immunohistochemistry}

Immunohistochemistry for smooth muscle actin (SMA) and vimentin was performed on $4 \mu \mathrm{m}$ sections and aminopropylethoxysilane-coated slides. In short, paraffin sections were deparaffinised and rehydrated. After antigen retrieval with citrate endogenous peroxidase was quenched with $\mathrm{H}_{2} \mathrm{O}_{2}$. Next, slides were washed in PBS and were incubated overnight at room temperature with a SMA-specific primary antibody diluted 1:80 (clone 1A4, concentration $100 \mu \mathrm{g} / \mathrm{ml}$, Cat. \# M0851, Dako, Glostrup, Denmark). Clone V9 and clone 1C7 were used as diluted culture supernatant (1:80 and 1:40) for detection of vimentin and keratin 13 expression, respectively (Cat. \# 2202MVI and Cat. \# 2202M1C7, EuroProxima B.V., Arnhem, the Netherlands). After washing with PBS sections were subjected to the standard three-step ABC method (Dako, Glostrup, Denmark). Immune complexes were visualized with diaminobenzidine (Sigma, St. Louis) in a Tris- $\mathrm{HCl}$ buffer containing $\mathrm{H}_{2} \mathrm{O}_{2}$. Mayer's hematoxylin was used for counterstaining. Brown staining of cytoplasm indicated positivity. Slides without primary antibody were used as a negative control.

\subsection{Flow cytometry and sorting}

During acquisition a gate was created to collect 20,000 keratin-positive single cell events using an LSRII (BD Biosciences, Erembodegem, Belgium) flow cytometer, equipped with a $21 \mathrm{~mW} 488 \mathrm{~nm}$ laser and a $20 \mathrm{~mW}$ $355 \mathrm{~nm}$ UV laser. A data file contained all events. The UV 355 BP 450/50-A/UV 355 BP 450/50-H vs. UV 355 BP 450/50-A pulse-processor was used to enrich for single cell events during acquisition and analysis. Detector $\mathrm{F}$, BLUE 488 BP 530/30-A and detector E, BLUE 488 BP 575/26-A, were used to collect FITC and RPE fluorescence, respectively.

For data analysis, DNA index (DI) and coefficient of variation $(\mathrm{CV})$ calculation the WinList 6.0 and ModFit 3.2.1 software packages were used (Verity Software House, Inc., Topsham, ME). N-color compensation was used for post-acquisition spectral cross-talk correction according to the manufacturer's instructions, without the use of hyperlog transformation or $\log$ bias.

To largely limit the effect of fragmented or damaged nuclei generated during sample preparation on genomic data sorting was restricted to the $\mathrm{G}_{0} \mathrm{G}_{1}$ vimentin-positive, keratin-negative stromal cell fractions and the vimentinnegative, keratin-positive tumour cell fractions. Cells were flow-sorted using a FACSAria I flow-sorter at 40 psi (BD Biosciences, Erembodegem, Belgium) with a $100 \mu \mathrm{m}$ nozzle at a frequency of approximately $52.4 \mathrm{kHz}$ in purity mode. The $488 \mathrm{~nm}$ and the $407 \mathrm{~nm}$ laser line were used for excitation. These settings allowed us to typically flow-sort $800 \times 10^{3}$ cells in $5 \mathrm{ml}$ FalconTM tubes. The following detector and filter settings were used during sorting: FITC fluorescence, detector E (BLUE 488 BP 530/30-A), RPE fluorescence, detector D, (BLUE 488 BP 575/26-A) and DAPI fluorescence, detector A (VIOLET 407 BP 450/50A). A dot plot VIOLET 407 BP 450/50-A/VIOLET 407 BP $450 / 50-\mathrm{H}$ vs. VIOLET $407 \mathrm{BP} 450 / 50-\mathrm{A}$ was created to gate out doublet and aggregates during sorting. After sorting, cells were centrifuged at $4,000 \times g$ for $10 \mathrm{~min}$ before DNA was extracted.

\subsection{DNA isolation}

DNA was isolated as described using an overnight digestion with proteinase $\mathrm{K}$ at $56^{\circ} \mathrm{C}$ [23]. DNA was purified using the Promega Protein Precipitation solution (Promega, Leiden, Netherlands, Cat. \# A1120) according to the manufacturer's instructions. DNA concentrations were determined using the Picogreen method (Invitrogen, Leiden, The Netherlands).

\subsection{SNP array analysis}

SNP arrays were performed at the Leiden Genome Technology Center (http://www.lgtc.nl) as described [16] with minor modifications: $0.4-1.0 \mu \mathrm{g}$ DNA was used as input in a multi-use activation step and was subsequently dissolved in $60 \mu \mathrm{l}$ resuspension buffer. Genotypes and the GCS score were extracted using Beadstudio 2.3 (Illumina, Eindhoven, The Netherlands). Only genotypes with a GCS over 0.5 were used to eliminate low quality calls from this analysis. LOH within a tumour sample was determined by comparison to the reference sample. Informative SNPs, SNPs at which the reference sample is heterozygous, were checked for homozygosity and LOH was indicated when two neighbouring informative SNPs were homozygous in the tumour sample.

The beadarraySNP package was adapted to combine copy number profiles, allele-specific intensities and the DNA index as described elsewhere [18]. 
In short, the lesser allele intensity ratio (LAIR) is calculated for all informative SNPs. This value is close to 1 when the contribution of both alleles of an SNP to the total intensity in the tumour is similar to that of the reference sample. The value is close to 0 when there is no signal for either of the alleles in the tumour (LOH). Allelic imbalances will show intermediate values. Regions with lesser allele intensity ratio close to 1 should have an even copy number. Next, a segmentation procedure is applied to find genomic regions that have the same copy number and LAIR [17]. The copy numbers assigned to each segment are adjusted to the measured DNA index obtained by flow cytometry. This is used to determine the copy number of both alleles in each genomic region referred to as the allelic state [18].

\subsection{Microsatellite analysis}

Genomic DNA was taken from 12 flow-sorted carcinomas samples that showed LOH on 3p14.2 (FHIT), 4p15.2 and/ or 6p21.3 (HLA-I) in the keratin-positive tumour fraction by SNP-array analysis. DNA extracted from the flow-sorted vimentin-positive, patient-matched normal endometrium fractions was used as reference. Ten informative microsatellite primer pairs were selected (for primer sequences: see Supplementary Table S1): D3S1313, D3S1234, D3S4103, D4S1609, D4S3013, D4S1546, D6S1666, D6S273, MOGC and D6S265 (Genome Database, http:// www.gdb.org). From each primer set the forward primer was labelled with FAM (keratin-positive fraction) or JOE (vimentin-positive fraction). The reverse primers were left unlabeled. Polymerase chain reactions (PCRs) were performed according to standard procedures (Supplementary Table S1). For each PCR ten nanogram of DNA was used as input. The fluorescent PCR products were run using an ABI 310 automated sequencer (Applied Biosystems, Foster City, USA) applying filter set F. The GeneScan $^{\mathrm{TM}}-500 \mathrm{ROX}^{\mathrm{TM}}$ was used as size standard (Applied Biosystems). The results were analyzed using the ABI prism GeneScan 3.1 program. All markers provided interpretable results.

\section{Results}

\subsection{Flow cytometry}

Flow cytometry is a reliable method to examine clonal expansion of cells in carcinoma samples. Based on DNA content inferences can be made about the contribution of different cell cycle phases and the DNA index (DI) of the tumour cells can be calculated.
After histogram analysis the average $\mathrm{CV}$ of the stromal cell fractions was $3.7 \%+/-1.2 \%$. Despite the good quality of the DNA histograms, cell populations with an abnormal DNA content were not detected in any of the 57 stromal cell fractions. DNA histograms from patient-matched normal endometrium or lymph nodes were used as unimodal controls. CVs were $3.3 \%+/-1.0 \%$ and $3.8 \%+/-1.3 \%$, respectively and in the same range as the carcinoma-stromal cell fractions. By flow cytometry no DNA aneuploidy was observed in these samples either. However, technically we were able to detect DNA aneuploidy in stromal fractions of cervical carcinomas, as demonstrated by a rare case (sample-58) containing poorly differentiated tumour cells (Supplementary Figure S1).

The percentage of cells in the S-phase of the tumour stromal cells, normal endometrium and lymph nodes were low and significantly lower $(p=0.0001)$ than the percentage of epithelial cells in the S-phase $(14.6 \%+/-8.1 \%)$ (Table 1).

Examples of DNA histograms with low background noise of the stromal compartments and of patient-matched normal endometrium are shown by Fig. 1 (sample-22 and sample-16, respectively). Using anti-keratin and antivimentin antibodies the epithelial fraction (red) and the stromal fraction (green) can be clearly separated (Fig. 1a and e). Unimodal DNA histograms were shown after gating on vimentin-positive cell fractions and were highly comparable with DNA histograms obtained from patientmatched controls in all cases. After gating on the keratinpositive fraction a DNA aneuploid tumour population was shown with a DI of 0.94 (Fig. 1d, sample-22). The second sample shows a DNA aneuploid population $(\mathrm{DI}=1.91)$ and a DNA pseudo-diploid population $(\mathrm{DI}=0.99)$ (Fig. 1h, sample-16).

In contrast, 14 (25\%) of the keratin-positive epithelial cell fractions were DNA pseudo-diploid. Forty three samples $(75 \%)$ showed a DNA aneuploid fraction. Ten of the DNA aneuploid samples were bimodal and also showed a second (minor) epithelial population, either DNA pseudo-diploid or DNA aneuploid (for details see Table 1).

\subsection{Confirmation of CAFs by IHC of SMA and vimentin}

To confirm the presence of CAFs in the samples, trimmed tissue sections were stained for SMA and vimentin by standard IHC. Figure 2 shows two examples of FFPE cervical carcinomas (A and B). The inter-epithelial stromal cells (S) show high SMA and high vimentin expression, whereas the tumour cells $(\mathrm{T})$ are negative. Some intratumour infiltrating cells were also stained by antivimentin. Twenty out of the 57 samples were selected that showed low numbers of infiltrating cells $(30 \%$ or lower) and high numbers $(>70 \%)$ of CAFs based on 
Table 1 Summary of flow cytometric analysis of cervical cancer stromal cells and epithelial cells, patient-matched normal endometrium and lymph nodes

\begin{tabular}{lcccc}
\hline Fraction & $\mathrm{V}+$ stroma & $\mathrm{K}+$ carcinoma & $\mathrm{V}+$ endometrium & $\mathrm{V}+$ lymph node \\
\hline Number & 57 & 57 & 57 & 57 \\
DNA (pseudo) diploid & 57 & 14 & 0 & 16 \\
DNA aneuploid (1 population) & 0 & 33 & 0 & 0 \\
DNA aneuploid and pseudo-diploid & 0 & 3 & 0 & 0 \\
DNA aneuploid (2 populations) & 0 & 7 & $3.3+/-1.0$ & 0 \\
Average\% CV+/- SD & $3.7+/-1.2$ & $4.1+/-1.0$ & $0.1+/-0.3$ & $3.8+/-1.3$ \\
Average\% S-phase +/- SD & $1.4+/-1.8$ & $14.6+/-8.5$ & $23.3+/-9.3$ & $0.6+/-1.0$ \\
Average\% BAD +/- SD & $14.1+/-7.7$ & $33.4+/-12.3$ & $12.1+/-9.1$ \\
\hline
\end{tabular}

DNA pseudo-diploid $0.95<$ DI $<1.05$

DNA aneuploid DI $\leq 0.95$ or DI $\geq 1.05$

$\mathrm{CV}=$ coefficient of variation

$\mathrm{BAD}=$ background, aggregates and debris of the DNA histogram calculated using ModFit

$\mathrm{SD}=$ standard deviation
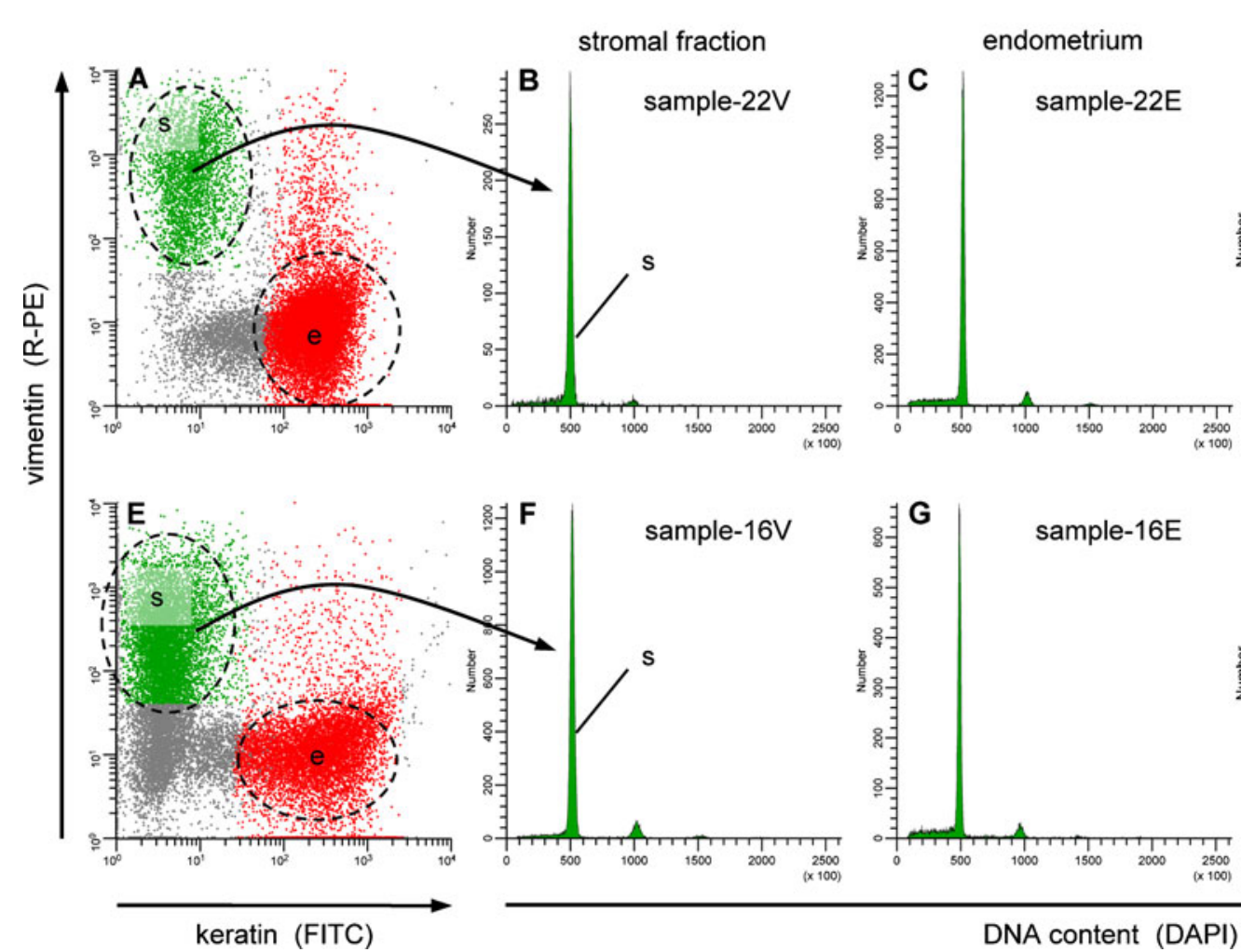

overlay stromal (s) / epithelial (e) fraction
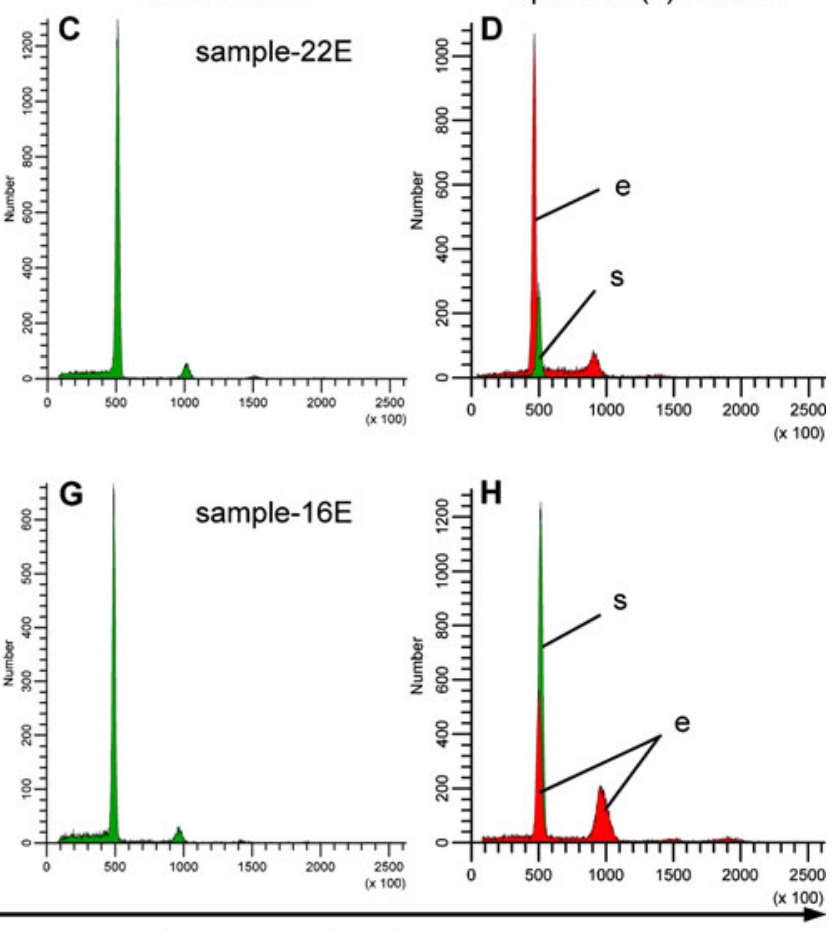

Fig. 1 Examples of attempts to detect DNA content abnormalities in the vimentin-positive, keratin-negative stromal cell fractions of FFPE cervical carcinomas by multi-parameter DNA flow cytometry. Cells isolated from $60 \mu \mathrm{m}$ sections after heat-induced antigen retrieval and enzymatic dissociation, were simultaneously labelled for keratin (FITC), vimentin (RPE) and DNA (DAPI) and measured using an LSRII flow cytometer. Patient-matched normal endometrium served as a DNA diploid reference. a) cervical carcinoma with one keratinpositive cycling population, e) cervical carcinoma with two keratinpositive cycling populations. After gating on the vimentin-positive, keratin-negative stromal cell fractions $(s$, green) unimodal DNA histograms are shown $(\mathbf{b}, \mathbf{f})$ and no DNA aneuploidy was detectable in these fractions, despite the good quality of the DNA histograms. DNA histograms of the stromal cell fractions were highly comparable with that of the endometrium (c, g) samples. In contrast, DNA aneuploidy could be readily detected in the vimentin-negative, keratinpositive epithelial (e) cell fractions. (d) A carcinoma keratin-positive DNA aneuploidy population with a DI of 0.94 . Note that some keratin-positive cells also express vimentin. (h) An epithelial cell fractions showing a bi-model DNA histogram with a DNA aneuploid population $(\mathrm{DI}=1.91)$ and a DNA pseudo-diploid population $(\mathrm{DI}=$ 0.99) (for LAIR analysis, see Supplementary Figure S3, sample-22E and $22 \mathrm{~V}$ and sample-16E and $16 \mathrm{~V}$, respectively) 

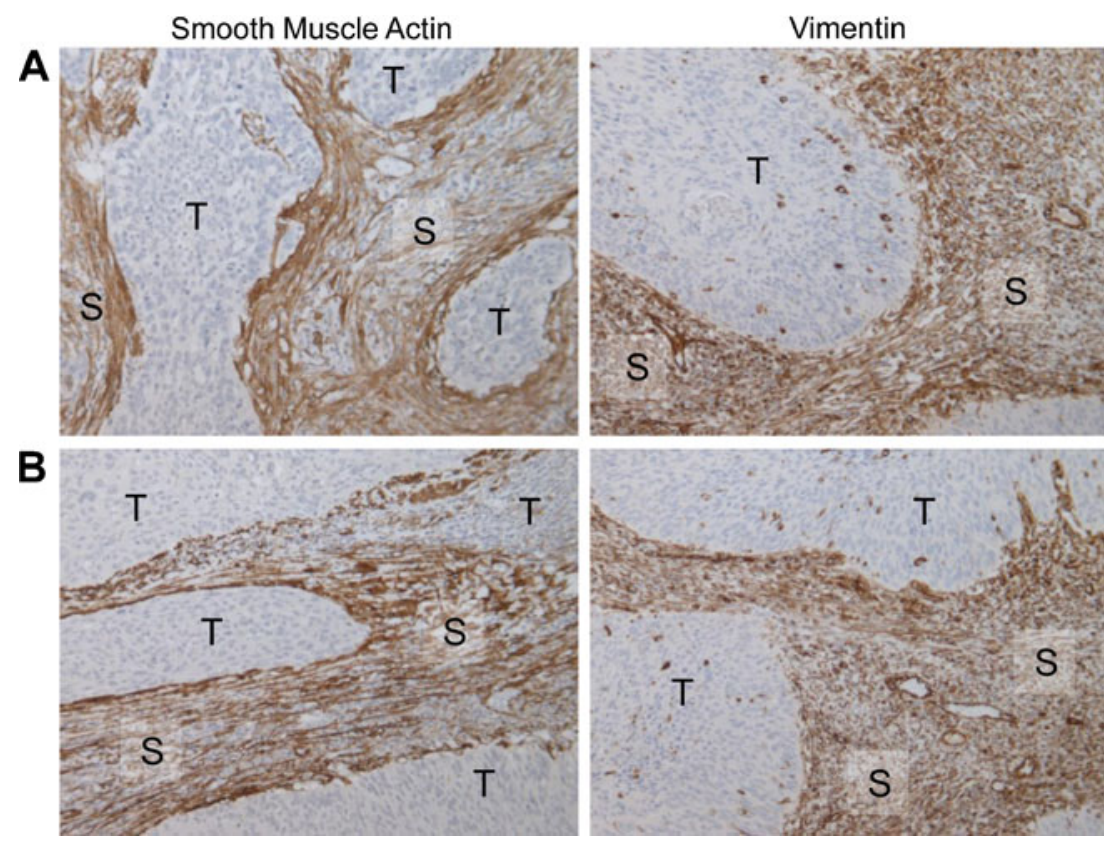

Fig. 2 Smooth muscle actin and vimentin are abundantly expressed in the stroma of cervical carcinoma. Smooth-muscle actin (SMA) and vimentin expression in tissue sections from cervical carcinomas showing high numbers of fibroblast neighbouring the carcinoma cells. a A DNA pseudo-diploid squamous cell carcinoma sample stained for smooth muscle actin (SMA) (left upper panel) and vimentin (right

smooth muscle actin (SMA) stained sections. These samples were initially processed for flow cytometry and sorting.

\subsection{Detection of CNAs and $\mathrm{LOH}$}

The $\mathrm{G}_{0} \mathrm{G}_{1}$ of the stromal cell fractions and the $\mathrm{G}_{0} \mathrm{G}_{1}$ of the epithelial cell fractions were flow-sorted. DNA was extracted and used in the GoldenGate 6K SNP-array. DNA extracted from flow-sorted vimentin-positive, keratin-negative histological tumour free patient-matched normal endometrium served as reference. Genome-wide genomic profiles from the flow-sorted stromal cells were compared with that of patientmatched controls and with the keratin-positive fractions.

The genotype concordance between the cervical cancer stromal cell fractions and the paired endometrial samples was $>99.9 \%$. By integrating the DNA index in the analysis true copy numbers can be estimated. This method was recently introduced as LAIR-analysis (lesser allele intensity ratio analysis) [18].

CNAs and LOH were not detected in any of the cervical cancer stromal cell fractions tested (Fig. 3). Examples of LAIR-analysis of the stromal compartments of two DNA aneuploid cervical carcinomas (sample-7 K, $\mathrm{DI}=0.92)$ and (sample-10 K, DI = 1.97) are shown in Fig. 3b and d (chromosomes 10-15, 21 only). In contrast, considerable numbers of CNAs and $\mathrm{LOH}$, as well as copy- upper panel). b A DNA aneuploid squamous cell carcinoma stained for SMA (lower left panel) and vimentin (lower right panel). In addition to vimentin these fibroblast also clearly express SMA as can be observed by standard IHC. This demonstrates that these cells resemble myofibroblasts (magnification $200 \times$ ). $\mathrm{t}=$ tumour, $\mathrm{s}=$ stroma

neutral $\mathrm{LOH}$, were detected in the keratin-positive cell fractions in all cases. Five examples of complete LAIRanalysis are shown in Supplementary Figure S2.

Since no somatic genetic alterations were identified in the stromal fractions of the selected tumours, we processed the remaining 37. Also in these samples no CNAs or LOH were detected (Supplementary Figure S3 shows LAIR-analysis of all stromal cell fractions and of all patient-matched endometrium cell fractions used in this study).

\subsection{Microsatellite analysis}

LAIR-analysis of the epithelial cell fractions showed that chromosomes $3 p, 4 p$ and $6 p$ were most often affected by absolute LOH. Losses were found in $47.4 \%, 40.5 \%$ and $45.4 \%$, of the cases, respectively. To confirm these findings we performed microsatellite analysis using informative markers (see Supplementary Table S1) on the flow-sorted epithelial cell fractions as well as on the stromal cell fractions and the normal patient-matched endometrium of 12 samples. LOH, detected in the epithelial cell fractions by LAIR-analysis, was confirmed by microsatellite analysis in all 12 samples tested. Figure 4 shows examples (A: sample10, B: sample 56 and C: sample-7, respectively) of results obtained using markers D3S1313, D4S1546 and D6S265. The flow-sorted epithelial cell fractions only show one allele. 

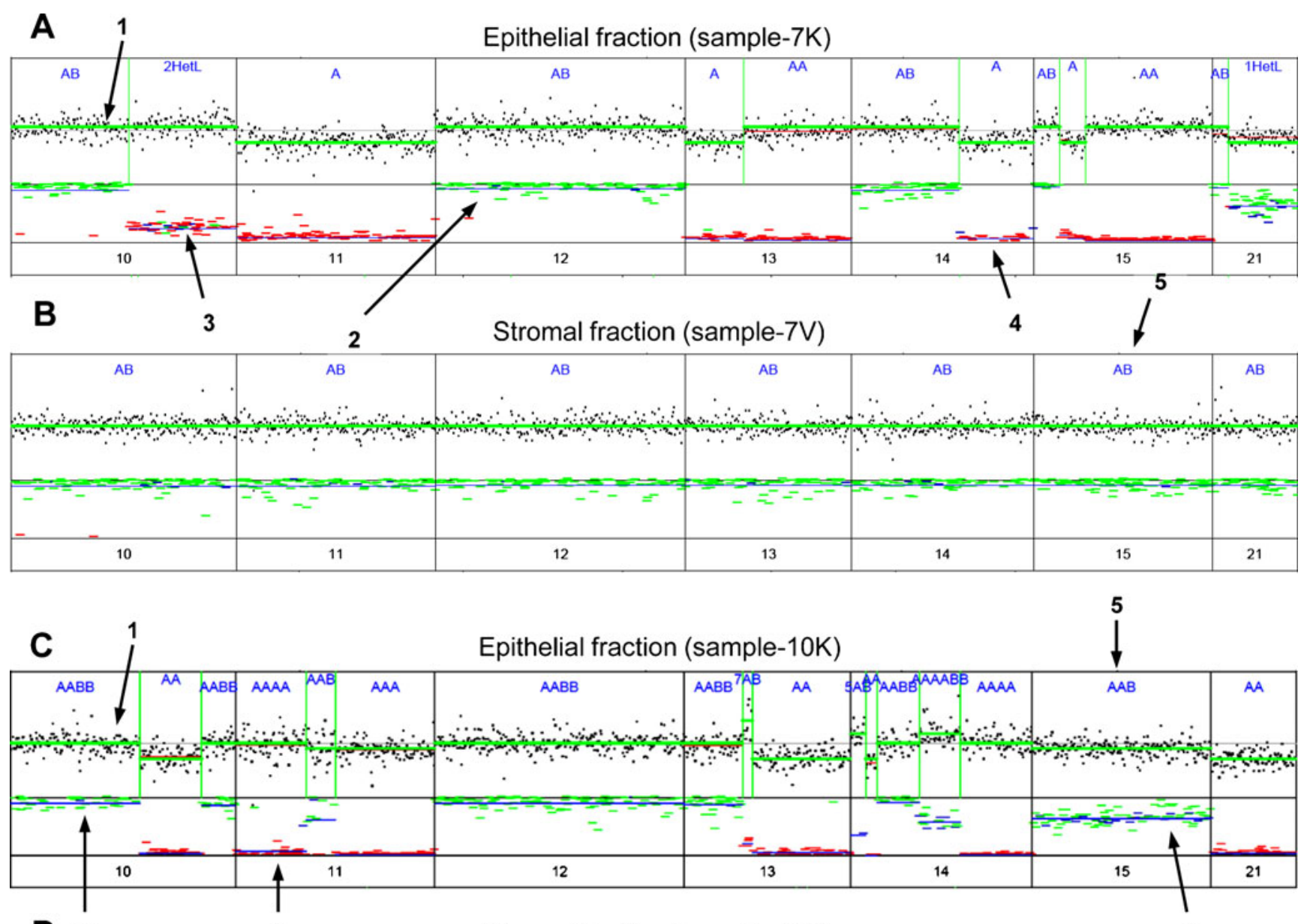

D 2

4

Stromal fraction (sample-10V)

3

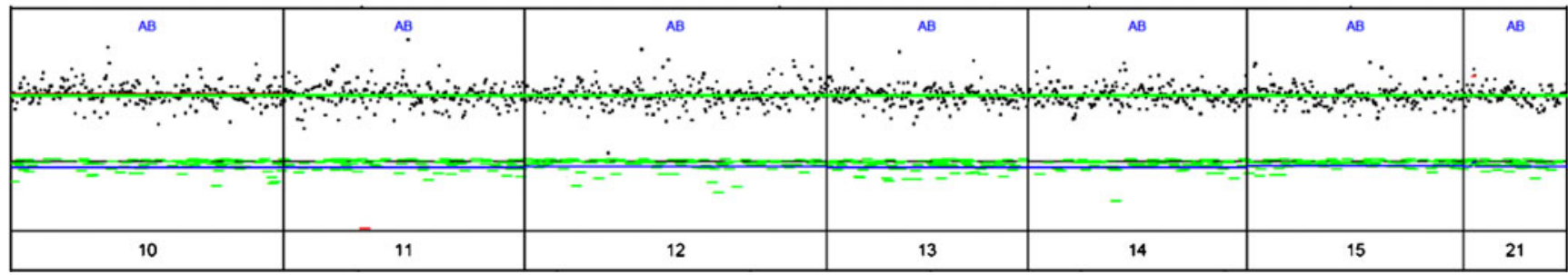

Fig. 3 LAIR analysis of cervical cancer stromal cells and epithelial cells. DNA extracted from flow-sorted cervical stromal cells with high percentage of CAFs and cervical carcinoma cells was analyzed on a $6 \mathrm{~K} \mathrm{SNP-array} \mathrm{highly} \mathrm{suitable} \mathrm{for} \mathrm{FFPE} \mathrm{tissues} \mathrm{(GoldenGate} \mathrm{assay,}$ Illumina). DNA from flow-sorted vimentin-positive endometrium cells served as a patient-matched control for genotyping. Representative LAIR plots of chromosomes $10-15$ and 21 are shown of the epithelial cell fraction and the stromal cell fraction of two DNA aneuploid cervical carcinomas: sample-7, DI $=0.92$ and sample-10, DI $=1.97$, respectively. Many alterations were identified in the epithelial cell fractions (see also Supplementary Figure S2). A, AB or AAB (characters in blue) represents the number of copies and the ratio between the alleles, respectively. LOH (red dotted lines) was detected on chromosomes 11, 13 and 14 in both samples (a, c). Note the copy neutral LOH on chromosomes 13 and 15 of sample-7 (a), relative to a DNA

In contrast, the tumour stromal cell fractions were identical to the vimentin-positive cell fractions of the endometrium and showed retention of heterozygosity for the tested loci in aneuploid background with a DI of 0.92, and on chromosomes 11 and 14 of sample-10 (c), relative to a highly DNA aneuploid background with a DI of 1.97. Allelic imbalances were detected on chromosomes 11,14 and 15 (AAB, AAAABB and AAB, respectively) of sample-10 (c). In sample-7 (a) a segment of chromosomes 10 and 21 could be observed that showed two or one copies while clearly showing an imbalance (2HetL and $1 \mathrm{HetL}$ ). This could be explained by a mixtures of $\mathrm{AB}$ and $\mathrm{AA}$, and $\mathrm{AB}$ and $\mathrm{A}$, respectively. In contrast, the vimentinpositive stromal cell fractions $(\mathbf{b}, \mathbf{d})$ of both examples show a normal heterozygous $(\mathrm{AB})$ genotype for all chromosomes and no CNAs and/or LOH can be detected (see also Supplementary Figure S3). black arrow $(1)=$ segmentation line (green); black arrow $(2)=$ heterozygous SNPs (green horizontal bars); black arrow (3) = allelic imbalance (mixture of green, blue and red bars); black arrow (4) = LOH (red bars); black arrow $(5)=$ allelic state $(\mathrm{AB}, \mathrm{AAB}, \mathrm{A}$, etc.)

all 12 cases as can be observed by presence of both alleles in the profiles (Fig. 4, compare the stromal/endometrium fractions with the epithelial fractions). 
epithelial fraction

stromal fraction

endometrium
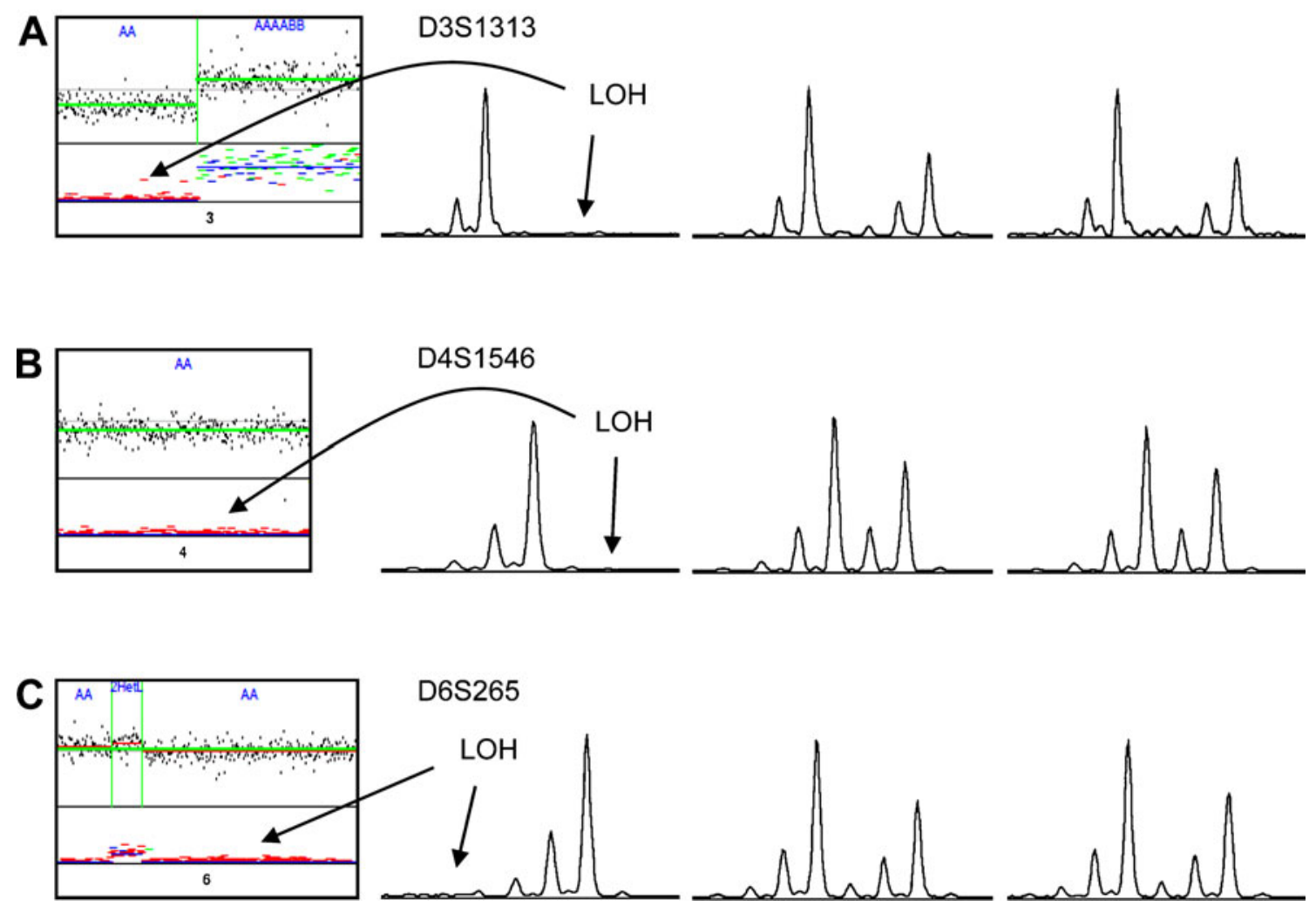

Fig. 4 Microsatellite analysis of chromosomes 3p, $4 p$ and $6 p$ in flowsorted cervical carcinoma stromal cells and epithelial cells. Figure shows results from two DNA aneuploid (a, b) one DNA pseudodiploid (c) FFPE cervical carcinoma. From sample-10, sample-56 and sample-7 LAIR plots are shown of chromosomes 3 (a), 4 (b) and 6 (c) demonstrating $\mathrm{LOH}$ of chromosomal segments. $\mathrm{LOH}$ on chromosome $6 p$ is copy neutral (c). The red vertical bars represent the position of the markers on the chromosomes. $\mathrm{LOH}$, as can be observed by loss of

\section{Discussion}

Using a combined approach of DNA content flow-sorting and SNP-arrays we found no evidence for somatic genetic alterations in stromal cell fractions from cervical carcinomas. We clearly show for the first time that these cell fractions are DNA diploid and hold a genotype that cannot be discriminated from patient-matched normal endometrium samples. From our study it can be concluded that somatic genetic alterations in cervical cancer tumour stroma cells do not occur and as a result cannot contribute to development and progression of cervical cancer.

Our findings are in contrast to several earlier studies in tumour stroma showing high frequencies of genomic aberrations, e.g. $\mathrm{LOH}$ and CNAs, in diverse types of carcinomas [12, 24-27]. Since LOH and CNAs are associated with DNA aneuploidy $[28,29]$ we should have been able to detect small DNA aneuploid populations in the stromal fractions using our multi-parameter flow cytometric technique. In spite of this 57 samples were devoid of DNA one of the alleles, was confirmed by microsatellite analysis using 3 to 4 markers (Supplementary Table S1) for each chromosomal segment. In contrast, no LOH was detected in the vimentin-positive, keratinnegative cell fractions of cervical carcinomas and patient-matched normal endometrium. Note the complete loss of the other allele in these samples demonstrating clonal expansion of these alterations and pure tumour populations due to FACS

aneuploidy in the stromal cell fractions. We added a sample containing a small fraction of poorly differentiated cells that lost keratin expression (Supplementary Figure S1) to show our technique is sensitive enough to pick up small subpopulations of aneuploid cells.

To our knowledge there are only a few other reports showing a lack of evidence of somatic genetic alterations in tumour stroma. These studies were all performed using microdissected stroma from fresh/frozen breast and ovarian cancer samples [30-33]. Conversely, the earlier studies that do show somatic genetic alterations in stroma used microdissected cells from FFPE archival samples. Hence, the somatic genetic changes found in CAFs might have been falsely introduced by the low yields and degraded DNA generally obtained after microdissection of formalin-fixed samples. Indeed, low concentrations of DNA input may cause spurious changes $[34,35]$. Degraded DNA has also been suggested as a source for errors [31, 33, 36]. However, in the current study we clearly demonstrated that with our preparation technique and FACS sufficient amounts of 
DNA can be isolated for SNP-array analysis from FFPE tissues without the introduction of artificial LOH or copy number changes due to low DNA yields or whole genome amplification [37]. In addition to the high cell yields that can be obtained after our collagenase/dispase dissociation method of FFPE tissues, FACS can separate tumour sub populations based on DNA index, allowing the study of intratumour heterogeneity [18, 38, 39]. Furthermore, restricting the sorting to the $\mathrm{G}_{0} \mathrm{G}_{1}$ population excludes damaged or fragmented nuclei from the tested DNA. This improves the quality of the genomic data generated from SNP-arrays (see Fig. 3). We also demonstrated for the first time that these kind of studies are possible in FFPE archival samples using multiparameter DNA flow-sorting and SNParrays. In retrospective studies typically only FFPE tissue is available, and it is often hard to obtain paired genetically normal tissue for comparison. FACS can overcome this limitation, and generates patient-matched tumour and control DNA where the handling and storage conditions of DNA from different cell populations have been identical.

Our findings match with the general paradigm of cervical carcinogenesis, that only the epithelial cell fraction of cervical carcinomas harbours HPV causing cellular and molecular alterations while the stromal cell fraction is free of this oncogenic virus [40]. As described by others epigenetic stromal changes seem to assist cervical cancer development and progression $[9,10]$. Amongst infiltrating lymphocytes showing tumour associated cytokine/chemokines repertoires, fibroblasts can be found that changed morphology and protein expression. Smooth muscle actin was abundantly expressed in the micro environment of the tumour nodules (see Fig. 2) reflecting the presence of myofibroblasts. Recent studies in oral squamous cell carcinoma also showed high levels of SMA expression [7], which was significantly associated with disease mortality [6]. In a previous study from our group we demonstrated high levels of (activated) TGF- $\beta$ in cervical cancer stroma [41]. Activated TGF- $\beta$ can explain the high expression of SMA, since this chemokine induces SMA expression [42]. Furthermore, TGF- $\beta$ is a potent inhibitor of cell cycle progression of normal cells [43], which can also explain the low percentage of stromal cells in S-phase comparable to S-phases found in normal endometrium and lymph nodes. These findings are similar to those observed in genetically unstable oral squamous cell carcinomas of which cultured CAFs showed less proliferative activity as compared to other fibroblast subtypes. Simultaneously, cell cycle-related genes were down-regulated [7]. Cancer cells can bypass a TGF- $\beta$ dependent cell cycle blockage. Indeed, a significantly higher average S-phase (average $15 \%$ ) was found for the epithelial cell fractions. Likely, CAFs do not proliferate locally but resemble a population of fibroblasts which proliferated and migrated from outside the tumour nodule and differentiated in myofibroblasts. Our observations are supported by a study into the origin of murine CAFs showing that at least $25 \%$ of the CAFs are generated in the bone marrow and migrate to the tumour stroma [4]. Taken together, these findings weaken the point of a local proliferation of the tumour micro environment and clonal outgrowth of stromal cells carrying somatic genetic changes $[12,13,44]$. The complete loss of an allele detected by microsatellite analysis is also hard to understand in light of the heterogeneous composition of the tumour stroma and microdissection as method of purification of cell populations. It can only be explained by a clonal expansion of a cell population devoid of unaffected cells. For isolation of DNA, tumour stroma nodules up to $5 \mathrm{~mm}$ distant from the tumour nodule were selected $[44,45]$. Tumour stroma is, in addition to fibroblast and CAFs, mainly composed of variable numbers of leukocytes. This is true not only in cervical but also in breast, colon, ovarian carcinomas and other types of cancer. The admixture of these (genetically normal) cells hinders the detection of absolute $\mathrm{LOH}$ resulting in measurements of allelic imbalances.

We are aware that the resolution of about $500 \mathrm{~kb}$ of the GoldenGate SNP-array used in our study is quite low. It is however sufficiently large to detect the reported whole chromosome arm losses or changes of multiple microsatellite markers in tumour stroma as described by other studies. Indeed, SNP-array analysis clearly showed (see Fig. 3) that all epithelial cell fractions, including those identified as DNA pseudo-diploid, harboured genetic alterations (manuscript in preparation). Microsatellite analysis confirmed the $\mathrm{LOH}$ identified using SNP-arrays at the cervical cancer hot spots on chromosomes $3 p$ [46-48], $4 p$ [49, 50] and $6 p$ [39, 46, 51] in the epithelial cells fractions but also did not reveal genetic alteration in any of the stromal cell populations tested. In addition, the epithelial fractions showed DNA aneuploidy in $75 \%$ of the samples, which is comparable to a multiparameter flow cytometric study using fresh/frozen samples. This demonstrates the reliability of our technique for FFPE samples [52].

In conclusion, we clearly show by DNA content flow cytometry, FACS and SNP-arrays that cervical cancer stromal cells are DNA diploid, are genetically stable and have a genotype undistinguishable from that of patientmatched normal endometrium. Our findings are of importance to the general understanding of cervical carcinogenesis. Stromal epigenetic alterations rather than stromal genetic alterations seem to play a role in cervical carcinogenesis. Furthermore, reliable genotypes can be generated from flowsorted tumour subpopulations of a large series of FFPE archival cervical carcinomas. In addition, the stromal compartment of cervical carcinomas can be used as DNA-matched control allowing large retrospective studies of archival FFPE tissues for which no normal reference tissue is available. 
Acknowledgements We thank Klaas van der Ham for his assistance in photography of tissue sections.

Disclosure/Conflict of Interest Potential conflicts do not exist for this study.

Author Contributions Willem Corver and Jan Oosting designed the study and wrote the paper. Willem Corver and Natalja ter Haar performed the experimental work including, cell preparation, FACS analysis and cell sorting, DNA extraction and purification, SNP genotyping, data analysis and immunohistochemistry. Natalja ter Haar performed microsatellite analysis. Gert Jan Fleuren reviewed all tissue sections and assisted in preparing the manuscript. Jan Oosting performed SNP-array analysis.

Open Access This article is distributed under the terms of the Creative Commons Attribution Noncommercial License which permits any noncommercial use, distribution, and reproduction in any medium, provided the original author(s) and source are credited.

\section{References}

1. N. Nakamura, T. Iijima, K. Mase, S. Furuya, J. Kano, Y. Morishita, M. Noguchi, Phenotypic differences of proliferating fibroblasts in the stroma of lung adenocarcinoma and normal bronchus tissue. Canc. Sci. 95, 226 (2004)

2. M. Hu, J. Yao, L. Cai, K.E. Bachman, F. van den Brule, V. Velculescu, K. Polyak, Distinct epigenetic changes in the stromal cells of breast cancers. Nat. Genet. 37, 899 (2005)

3. A. Orimo, P.B. Gupta, D.C. Sgroi, F. Arenzana-Seisdedos, T. Delaunay, R. Naeem, V.J. Carey, A.L. Richardson, R.A. Weinberg, Stromal fibroblasts present in invasive human breast carcinomas promote tumor growth and angiogenesis through elevated SDF-1/ CXCL12 secretion. Cell 121, 335 (2005)

4. N.C. Direkze, K. Hodivala-Dilke, R. Jeffery, T. Hunt, R. Poulsom, D. Oukrif, M.R. Alison, N.A. Wright, Bone marrow contribution to tumor-associated myofibroblasts and fibroblasts. Canc. Res. 64, $8492(2004)$

5. A.E. Karnoub, A.B. Dash, A.P. Vo, A. Sullivan, M.W. Brooks, G. W. Bell, A.L. Richardson, K. Polyak, R. Tubo, R.A. Weinberg, Mesenchymal stem cells within tumour stroma promote breast cancer metastasis. Nature 449, 557 (2007)

6. D. Marsh, K. Suchak, K.A. Moutasim, S. Vallath, C. Hopper, W. Jerjes, T. Upile, N. Kalavrezos, S.M. Violette, P.H. Weinreb, K.A. Chester, J.S. Chana, J.F. Marshall, I.R. Hart, A.K. Hackshaw, K. Piper, G.J. Thomas, Stromal features are predictive of disease mortality in oral cancer patients. J. Pathol. 223, 470 (2011)

7. K.P. Lim, N. Cirillo, Y. Hassona, W. Wei, J.K. Thurlow, S.C. Cheong, G. Pitiyage, E.K. Parkinson, S.S. Prime, Fibroblast gene expression profile reflects the stage of tumour progression in oral squamous cell carcinoma. J. Pathol. 223, 459 (2011)

8. G. Finak, N. Bertos, F. Pepin, S. Sadekova, M. Souleimanova, H. Zhao, H. Chen, G. Omeroglu, S. Meterissian, A. Omeroglu, M. Hallett, M. Park, Stromal gene expression predicts clinical outcome in breast cancer. Nat. Med. 14, 518 (2008)

9. D. Gius, M.C. Funk, E.Y. Chuang, S. Feng, P.C. Huettner, L. Nguyen, C.M. Bradbury, M. Mishra, S. Gao, B.M. Buttin, D.E. Cohn, M.A. Powell, N.S. Horowitz, B.P. Whitcomb, J.S. Rader, Profiling microdissected epithelium and stroma to model genomic signatures for cervical carcinogenesis accommodating for covariates. Canc. Res. 67, 7113 (2007)

10. S. Sahebali, G. Van den Eynden, E.F. Murta, M.A. Michelin, P. Cusumano, P. Petignat, J.J. Bogers, Stromal issues in cervical cancer: a review of the role and function of basement membrane, stroma, immune response and angiogenesis in cervical cancer development. Eur. J. Canc. Prev. 19, 204 (2010)

11. M. Allen, J.J. Louise, Jekyll and Hyde: the role of the microenvironment on the progression of cancer. J. Pathol. 223, $162(2011)$

12. F. Moinfar, Y.G. Man, L. Arnould, G.L. Bratthauer, M. Ratschek, F.A. Tavassoli, Concurrent and independent genetic alterations in the stromal and epithelial cells of mammary carcinoma: implications for tumorigenesis. Canc. Res. 60, 2562 (2000)

13. K. Fukino, L. Shen, A. Patocs, G.L. Mutter, C. Eng, Genomic instability within tumor stroma and clinicopathological characteristics of sporadic primary invasive breast carcinoma. JAMA 297, $2103(2007)$

14. K. Ishiguro, T. Yoshida, H. Yagishita, Y. Numata, T. Okayasu, Epithelial and stromal genetic instability contributes to genesis of colorectal adenomas. Gut 55, 695 (2006)

15. H. Yagishita, T. Yoshida, K. Ishiguro, Y. Numata, I. Okayasu, Epithelial and stromal genetic instability linked to tumor suppressor genes in ulcerative colitis-associated tumorigenesis. Scand. J. Gastroenterol. 43, 559 (2008)

16. E.H. Lips, J.W. Dierssen, R. van Eijk, J. Oosting, P.H. Eilers, R.A. Tollenaar, E.J. de Graaf, R. van't Slot, C. Wijmenga, H. Morreau, T. van Wezel, Reliable high-throughput genotyping and loss-ofheterozygosity detection in formalin-fixed, paraffin-embedded tumors using single nucleotide polymorphism arrays. Canc. Res. 65, 10188 (2005)

17. J. Oosting, E.H. Lips, R. van Eijk, P.H. Eilers, K. Szuhai, C. Wijmenga, H. Morreau, T. van Wezel, High-resolution copy number analysis of paraffin-embedded archival tissue using SNP BeadArrays. Genome Res. 17, 368 (2007)

18. W.E. Corver, A. Middeldorp, N.T. Ter Haar, E.S. Jordanova, M. van Puijenbroek, R. van Eijk, C.J. Cornelisse, G.J. Fleuren, H. Morreau, J. Oosting, T. van Wezel, Genome-wide allelic state analysis on flow-sorted tumor fractions provides an accurate measure of chromosomal aberrations. Canc. Res. 68, 10333 (2008)

19. P.S. Rabinovitch, Practical considerations for DNA content and cell cycle analysis, in Clinical flow cytometry, ed. by K.D. Bauer, R.E. Duque, T.V. Shankey (Williams \& Wilkins, Baltimore, 1993), p. 117

20. W.E. Corver, N.T. Ter Haar, E.J. Dreef, N.F. Miranda, F.A. Prins, E.S. Jordanova, C.J. Cornelisse, G.J. Fleuren, High-resolution multi-parameter DNA flow cytometry enables detection of tumour and stromal cell subpopulations in paraffin-embedded tissues. J. Pathol. 206, 233 (2005)

21. W.E. Corver, N.T. Ter Haar, High-resolution multiparameter DNA flow cytometry for the detection and sorting of tumor and stromal subpopulations from paraffin-embedded tissues. Curr. Protoc. Cytom. Chapter 7, Unit 7.37 (2011)

22. J.K. Glogovac, P.L. Porter, D.E. Banker, P.S. Rabinovitch, Cytokeratin labeling of breast cancer cells extracted from paraffin-embedded tissue for bivariate flow cytometric analysis. Cytometry 24, 260 (1996)

23. E.C.A. Abeln, W.E. Corver, N.J. Kuipers-Dijkshoorn, G.J. Fleuren, C.J. Cornelisse, Molecular genetic analysis of flowsorted ovarian tumour cells: Improved detection of loss of heterozygosity. Br. J. Canc. 70, 255 (1994)

24. R.F. Paterson, T.M. Ulbright, G.T. MacLennan, S. Zhang, C.X. Pan, C.J. Sweeney, C.R. Moore, R.S. Foster, M.O. Koch, J.N. Eble, L. Cheng, Molecular genetic alterations in the laser-capturemicrodissected stroma adjacent to bladder carcinoma. Cancer 98, $1830(2003)$ 
25. N. Matsumoto, T. Yoshida, K. Yamashita, Y. Numata, I. Okayasu, Possible alternative carcinogenesis pathway featuring microsatellite instability in colorectal cancer stroma. Br. J. Canc. 89, 707 (2003)

26. K. Fukino, L. Shen, S. Matsumoto, C.D. Morrison, G.L. Mutter, C. Eng, Combined total genome loss of heterozygosity scan of breast cancer stroma and epithelium reveals multiplicity of stromal targets. Canc. Res. 64, 7231 (2004)

27. F. Weber, Y. Xu, L. Zhang, A. Patocs, L. Shen, P. Platzer, C. Eng, Microenvironmental genomic alterations and clinicopathological behavior in head and neck squamous cell carcinoma. JAMA 297, 187 (2007)

28. A.J. Holland, D.W. Cleveland, Boveri revisited: chromosomal instability, aneuploidy and tumorigenesis. Nat. Rev. Mol. Cell Biol. 10, 478 (2009)

29. T. Ried, Homage to Theodor Boveri (1862-1915): Boveri's theory of cancer as a disease of the chromosomes, and the landscape of genomic imbalances in human carcinomas. Environ. Mol. Mutagen. 50, 593 (2009)

30. M. Allinen, R. Beroukhim, L. Cai, C. Brennan, J. Lahti-Domenici, H. Huang, D. Porter, M. Hu, L. Chin, A. Richardson, S. Schnitt, W.R. Sellers, K. Polyak, Molecular characterization of the tumor microenvironment in breast cancer. Cancer Cell 6, 17 (2004)

31. W. Qiu, M. Hu, A. Sridhar, K. Opeskin, S. Fox, M. Shipitsin, M. Trivett, E.R. Thompson, M. Ramakrishna, K.L. Gorringe, K. Polyak, I. Haviv, I.G. Campbell, No evidence of clonal somatic genetic alterations in cancer-associated fibroblasts from human breast and ovarian carcinomas. Nat. Genet. 40, 650 (2008)

32. A.N. Hosein, M. Wu, S.L. Arcand, S. Lavallee, J. Hebert, P.N. Tonin, M. Basik, Breast carcinoma-associated fibroblasts rarely contain p53 mutations or chromosomal aberrations. Canc. Res. 70, 5770 (2010)

33. I. Campbell, W. Qiu, I. Haviv, Genetic changes in tumour microenvironments. J. Pathol. 223, 450 (2011)

34. T.G. Paulson, P.C. Galipeau, B.J. Reid, Loss of heterozygosity analysis using whole genome amplification, cell sorting, and fluorescence-based PCR. Genome Res. 9, 482 (1999)

35. N.L. Sieben, N.T. Ter Haar, C.J. Cornelisse, G.J. Fleuren, A.M. Cleton-Jansen, PCR artifacts in LOH and MSI analysis of microdissected tumor cells. Hum. Pathol. 31, 1414 (2000)

36. I. Campbell, K. Polyak, I. Haviv, Clonal mutations in the cancerassociated fibroblasts: the case against genetic coevolution. Canc. Res. 69, 6765 (2009)

37. E. Arriola, M.B. Lambros, C. Jones, T. Dexter, A. Mackay, D.S. Tan, N. Tamber, K. Fenwick, A. Ashworth, M. Dowsett, J.S. Reis-Filho, Evaluation of Phi29-based whole-genome amplification for microarray-based comparative genomic hybridisation. Lab. Investig. 87, 75 (2007)

38. W.E. Corver, L.A. Koopman, J. Van der Aa, M. Regensburg, G.J. Fleuren, C.J. Cornelisse, Four-color multiparameter DNA flow cytometric method to study phenotypic intratumor heterogeneity in cervical cancer. Cytometry 39, 96 (2000)

39. L.A. Koopman, W.E. Corver, A.R. Van der Slik, M.J. Giphart, G.J. Fleuren, Multiple genetic alterations cause frequent and heterogeneous human histocompatibility leukocyte antigen class I loss in cervical cancer. J Exp Med 191, 961 (2000)

40. M.A. de Boer, E.S. Jordanova, G.G. Kenter, A.A. Peters, W.E. Corver, J.B. Trimbos, G.J. Fleuren, High human papillomavirus oncogene mRNA expression and not viral DNA load is associated with poor prognosis in cervical cancer patients. Clin. Canc. Res. 13, 132 (2007)

41. S. Hazelbag, A. Gorter, G.G. Kenter, L. Van den Broek, G. Fleuren, Transforming growth factor-betal induces tumor stroma and reduces tumor infiltrate in cervical cancer. Hum. Pathol. 33, 1193 (2002)

42. V.V. Petrov, R.H. Fagard, P.J. Lijnen, Stimulation of collagen production by transforming growth factor-betal during differentiation of cardiac fibroblasts to myofibroblasts. Hypertension 39, $258(2002)$

43. J. Massagué, S.W. Blain, R.S. Lo, TGFb signaling in growth control, cancer, and heritable disorders. Cell 103, 295 (2000)

44. F. Weber, L. Shen, K. Fukino, A. Patocs, G.L. Mutter, T. Caldes, C. Eng, Total-genome analysis of BRCA1/2-related invasive carcinomas of the breast identifies tumor stroma as potential landscaper for neoplastic initiation. Am. J. Hum. Genet. 78, 961 (2006)

45. A. Patocs, L. Zhang, Y. Xu, F. Weber, T. Caldes, G.L. Mutter, P. Platzer, C. Eng, Breast-cancer stromal cells with TP53 mutations and nodal metastases. N. Engl. J. Med. \%20(357), 2543 (2007)

46. J.S. Rader, D.S. Gerhard, M.J. O'Sullivan, Y. Li, L. Li, H. Liapis, P.C. Huettner, Cervical intraepithelial neoplasia III shows frequent allelic loss in 3p and 6p. Gene Chromosome Canc. 22, 57 (1998)

47. F. Luft, J. Gebert, A. Schneider, P. Melsheimer, D.M. von Knebel, Frequent allelic imbalance of tumor suppressor gene loci in cervical dysplasia. Int. J. Gynecol. Pathol. 18, 374 (1999)

48. C.H. Choi, K.M. Lee, J.J. Choi, T.J. Kim, W.Y. Kim, J.W. Lee, S. J. Lee, J.H. Lee, D.S. Bae, B.G. Kim, Hypermethylation and loss of heterozygosity of tumor suppressor genes on chromosome $3 p$ in cervical cancer. Canc. Lett. 255, 26 (2007)

49. J.B. Sherwood, N. Shivapurkar, W.M. Lin, R. Ashfaq, D.S. Miller, A.F. Gazdar, C.Y. Muller, Chromosome 4 deletions are frequent in invasive cervical cancer and differ between histologic variants. Gynecol. Oncol. 79, 90 (2000)

50. R.K. Singh, D. Indra, S. Mitra, R.K. Mondal, P.S. Basu, A. Roy, S. Roychowdhury, C.K. Panda, Deletions in chromosome 4 differentially associated with the development of cervical cancer: evidence of slit2 as a candidate tumor suppressor gene. Hum. Genet. 122, 71 (2007)

51. C.F. Vermeulen, E.S. Jordanova, Y.A. Zomerdijk-Nooijen, N.T. Ter Haar, A.A. Peters, G.J. Fleuren, Frequent HLA class I loss is an early event in cervical carcinogenesis. Hum. Immunol. 66, 1167 (2005)

52. R. Kimmig, T. Kapsner, H. Spelsberg, M. Untch, H. Hepp, DNA cellcycle analysis of cervical cancer by flow cytometry using simultaneous cytokeratin labelling for identification of tumour cells. J. Canc. Res. Clin. Oncol. 121, 107 (1995) 